\author{
Military Technical College \\ Kobry El-Kobbah, \\ Cairo, Egypt
}

\author{
$14^{\text {th }}$ International Conference on \\ Applied Mechanics and \\ Mechanical Engineering.
}

\title{
Feature recognition of prismatic parts for inspection planning
}

By

Hesham A.Hussien*

Amro M.Youssef ${ }^{\star}$

Mohamed K.Shoukrey**

\begin{abstract}
:
Automated feature recognition has recognized as the front end of fully automated computer aided process planning (CAPP) systems. Automated feature recognition is considered to be the link between computer aided design (CAD) and CAPP systems. Feature recognition converts the geometrical and topological data contained in CAD file into application orientated features for planning purposes. This work introduces an automated rule based feature recognition algorithm to extract prismatic features from a boundary representation (Brep) solid model. The automatic feature recognition algorithm is developed using ACIS geometric kernel and $\mathrm{C}_{++}$object oriented programming. The recognition system input is a $\mathrm{B}$ rep solid model in sat format. The developed algorithm is a part of a complete computer aided inspection planning system.
\end{abstract}

\section{Keywords:}

CAD, CAD/CAM, CAPP, Feature recognition and Feature extraction 
* Egyptian Armed Forces

** College of Engineering, GUC, Cairo, Egypt

\section{Introduction:}

Process planning is defined as the efficient methods used to extract (decompose) features from CAD database for downstream applications [1]. Process planning activities can be done either in conventional way (Human based) or in computer assisted way. The human based planning is done using the manuals and self experience of the operator. The human based technique has the following problems, [1]:

1. Accumulation of experience need long time to be done.

2. The experience represents only approximate knowledge.

3. The Experience cannot deal with new processes or new systems.

Computer aided process planning (CAPP) is a computer assisted way that enables the encoding of human experiences into computer programs and building up knowledge and stating different rules that enable computers to deal with new products.

There are three general Geometrical Data input formats to any process plan. They are the code format, the text format and the graphical format. The code format is used with the variant approach where the part is represented by a code to retrieve its part family's plan. The text format can be in the form of some questions answered in an interactive environment using the user input. The graphical format is the part's CAD solid model. CAD models contain low level information while downstream activities require high level one. The link between CAD model and CAPP activities is done manually or by automated feature recognition systems. Automated feature recognition systems enable inspection planning and Computer Aided Manufacturing (CAM) operations to proceed automatically without human guidance or intervention [2]. Features have been considered as the technology that bridges the gap or as the communication medium between CAD and any planning application. Feature definitions are application dependant but generally, features are defined as geometric and topological patterns of interest in a part model.

For designers, a feature presents functionality while for machinists, a feature attempts to capture the effect of a cutting operation whilst for assembly planners, a feature represents a region of a part which will mate or connect with a corresponding feature on other part at the same time for inspection planner, a feature represents a pattern of measurement points [3]. Feature gives a higher conceptual meaning characteristic by dissecting component geometry into recognizable and meaningful forms [4].

The two major approaches in feature technology are the design by feature approach and the feature recognition approach. In design by feature approach, the model itself is generated using pre-defined features. Due to the different restrictions and limitations applied on the designers using the design by features approach, the feature recognition approach appears to be more generic and effective. Feature recognition is a sub-discipline of solid modeling that focuses on the design and implementation of algorithms for detecting functional information from solid models. 
The automation and creation of feature recognition algorithms is done by using object oriented programming (OOP) Techniques. The development of feature recognition algorithms using OOP to extract included information in any CAD file data base is a preparatory step for any CAPP system. Among the different part representation schemes and databases, Boundary representation (B-rep) appeared as the most suitable representation for automated feature recognition systems.

Feature Recognition from solid models has been a subject of research since 1980s [3,5]. Many different feature recognition approaches was reported by liu, et al [6], as follows:

1. 2D Sectioning.

2. Entity growing.

3. Syntactic pattern recognition.

4. Traversing CSG trees.

5. Volumetric decomposition (convex-Hull and cell composition).

6. Matching algorithms (Graph-based and Hint-based).

7. Geometric reasoning (Rule-based).

Other recognition methods were developed based on the orthographic projection by Lee, et al [7] or the neural network approach by Chang [8].

2D Sectioning and volumetric decomposition approaches are classified by shah [9] as machining region recognition in which machining volume to be removed is recognized instead of design, form and functional features. Other approaches differ from machining volume recognition approaches in that portions of geometric model are compared with predefined generic features to identify instances that match the predefined ones. Predefined generic features can be on of the following, an adjacency graph as in graph based approach, series of lines and curves as in syntactic pattern recognition approach or set of rules as in rulebased systems.

Traversing constructive solid geometry (CSG) trees approach has limited contributions to feature recognition. This approach has a major problem that CSG trees are not uniquely representing an object [9].

Babic, et al [10] introduced the potentials and limitations of the above mentioned feature recognition approaches.

The geometric reasoning and rule-based systems use geometry and topology for recognizing features. The rule-based approaches are the most recent and generic technique for feature recognition. These systems are well interfaced with different neutral formats such as IGES and STEP using the B-rep solid model.

This work will develop an automated rule-based feature recognition algorithm using ACIS geometric kernel and $\mathrm{C}_{++}$object oriented programming. The feature recognition system can recognize prismatic features based on planner and cylindrical surfaces. The system input is a B-rep solid model given in SAT format. This work is a part of a complete computer aided inspection planning project.

\section{Feature recognition algorithm:}

In this work, the feature recognition algorithm starts by inspecting the given B-rep solid model. The recognition algorithm extensively reason about both the geometry and the topology of the 
input solid model. It uses different transformations and membership relations to recognize high level features. The recognition algorithm is independent of the modeling technique used during creating the part.

Plane faces, slots, complete and incomplete cylindrical holes and shafts are extracted in this work. Depending on the application, other geometric features can be included in the extraction algorithm. Some rules are stated to define the extracted features. These rules are expressed in logic statements using object orientated programming. The recognition algorithm starts the by reading a given CAD file in SAT format using ACIS and $\mathrm{C}_{++}$. The developed algorithm loads the included entity list inside the SAT file. Starting from the face list that forming the target test piece and using $\mathrm{C}++$ functions, a search process extracts the included face list of the input B-rep model. This list is then classified into three separate lists. The first list is the plan faces list which contains all the planner faces in the body. The second list is the conical faces list which contains all the cylindrical and tapered surfaces. The third list is for spherical and spline surfaces. This list can be created for the inspection of free form surfaces. For the purpose of inspecting geometric primitives used in three axes machining, only the first two lists are used in this work. The plan faces list is used to search for any high level features that is based on planner surfaces such as pockets, steps and slots. An additional information list is developed for normal vectors of the plane faces exist in plan faces list. In B-rep, the normal vector is always pointing outward the material side to the air side. This allows the probe to approach the face from the direction opposite to the material side.

\subsection{Slot features recognition:}

Slot features are example of features that based on planner surfaces. It is agreed that the slot consists of three planner faces; one of them intersects the other two faces and is called the base. This three faces should satisfy some conditions to form a slot feature.

With the help of Figure (1), There may be some ambiguity in recognizing that three planer faces will form a slot. These cases may be summarized as follows:

1. Three planner faces each one intersects the others (e.g. F5, F6 and F7).

2. Three planner faces with one called the base intersects the two sides but the two side's normal vectors are in the same direction (e.g. F1, F3 and F2 as a base).

3. Three planner faces with one called the base intersects the two sides and the two side's normal vector are in opposite directions but forming a boss instead of a slot (e.g. F4, F6 and F5 as a base).

4. Sides that have normal vectors that are perpendicular (e.g. F2, F5 and F7 as a base).

As a result, The following rules are stated to guarantee that 3 planner faces form a slot feature:

1. Three planner faces with only one face (called the "base") intersects the other two faces (called the "sides").

2. Two side's normal vectors are in opposite directions.

3. There is no material exists between the side faces.

4. Slot base face must lie in between the side faces.

These conditions (rules) are coded in an object oriented programming statements. The set of plan faces that conform to these stated and coded rules are stored as a slot feature object. 


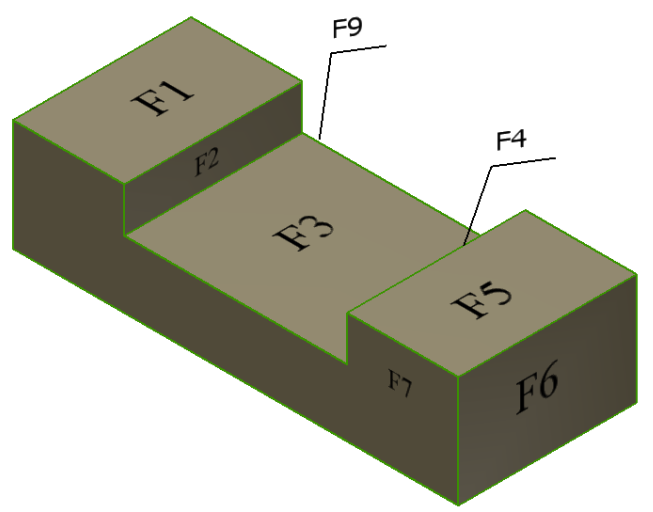

Figure (1): Slot Feature

The recognition algorithm searches for any three faces conform to the above stated rules by creating a loop that traverses heuristically through the planner faces list. Each three adjacent faces in the list are defined as a face group. The face groups under investigation are sent to form new list of potential slot candidates in order to not alter the original list.

An intersection test is applied to all face groups in the new list to guarantee that there is only one face intersects the others (condition no.1). This face is called the base and others called the sides. The face group is ordered such that the first face in the group is always the group base and the next two faces are the group sides. The side faces are then tested for normal vectors condition (condition no. 2).

The face groups are then tested for the material existence between the side faces (condition no.3). This test results in rejecting face groups forming bosses instead of slot features. This is checked by scalar product between normal vector of one side and the vector directed from this side to the other one. The scalar product result is greater than zero if the tested group forms a slot feature. The scalar product iless than zero if the tested group forms a boss, figure (2).
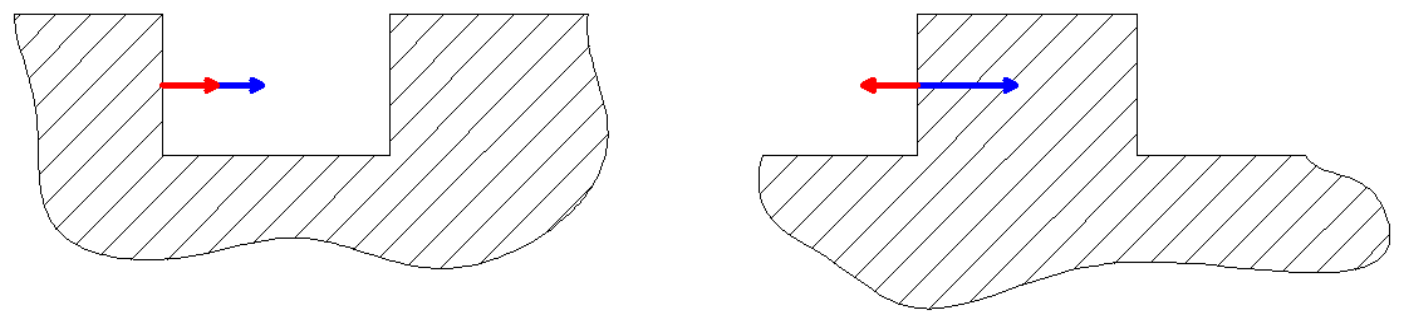

Figure (2): Material existence test

After applying the first three stated conditions, a slot like feature may pass. This can be demonstrated by, the face groups F7 as a slot base with F2 and F4 as a slot sides and F9 as a slot base with F2 and F4 as a slot sides, figure (1). These groups are not a real slot although they satisfy stated conditions. To avoid this case, a projection based methodology and a membership relation is investigated to check the existence of the slot base in between the slot sides (condition no. 4) (as in case of the face group formed by F2, F3 and F4 as shown in figure (1)). The methodology is based on creating a point above the slot base in the direction of the base normal vector by a distance equals to $0.1 \mu \mathrm{m}$. the test point is then projected onto the two slot sides. The slot sides are defined using a point on the side face and 
the side's normal vector. After the projection on the side faces, its membership relation to the side faces is tested. In case of face group such as (F2, F4 and F7), figure(3), the projected points will lie out of the side faces while in case of real slot that is formed by face group (F2, F4 and F3), figure (3), the projected point will lie in the side faces. It is important to be mentioned that the projection direction is the negative to the side faces normal vectors.

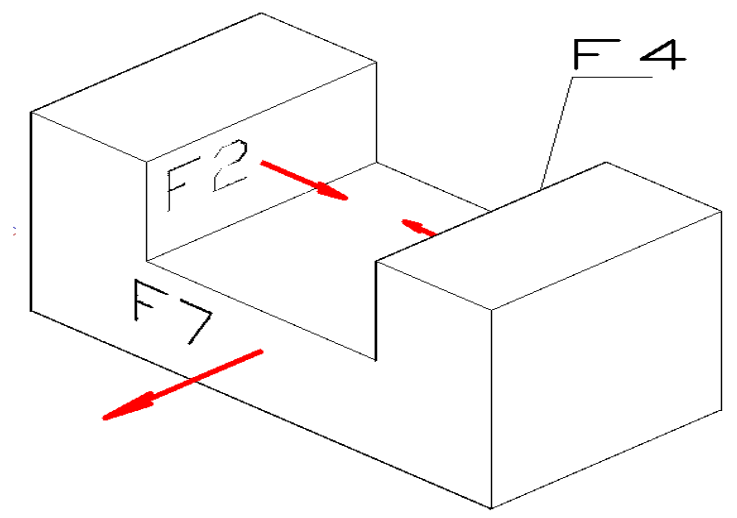

Figure (3): Face group that fails in the projection test

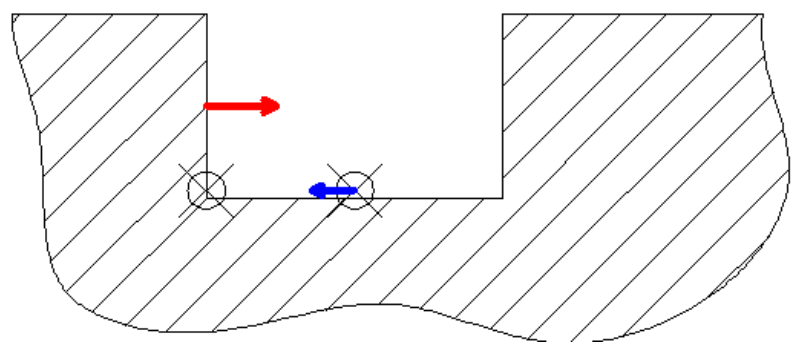

Figure (4): Face group that passes the projection test

Once a real slot feature is discovered, it is stored separately as a slot feature object and is then added to the slot features dynamic list. Each discovered slot feature is assigned with an ID number. The slot feature includes pointers to its base, side faces and its ID number.

\subsection{Cylindrical hole feature recognition:}

Cylindrical hole features are an example of features based on conical faces. Conical features are two types of surface geometries: tapered features or constant diameter features as machined holes. Constant diameter conical features are focused upon. Cylindrical surfaces are extracted from the original conical face list using geometric reasoning methods. Different geometrical parameters associated with cylindrical faces are automatically extracted from the solid model. These geometrical parameters are cylindrical face major axis direction, center point and radius. Cylindrical surfaces may form a hole feature or a shaft feature.

Scalar product between a surface point normal vector and a vector directed form this surface point to a point on the cylindrical axis (in the same plane) is used to classify the cylindrical face as a hole or a shaft as shown in figure (5). These vectors are calculated by using the geometrical parameters of a surface and a center points lies in the same plane. If the scalar product is greater than zero, a hole feature is recognized. If the scalar product is smaller than 
zero a shaft feature is recognized. As seen in figure (5), Difference between hole and shaft features based on cross product between a surface point normal (gray vector) and a vector from the surface point to center point (black vector).
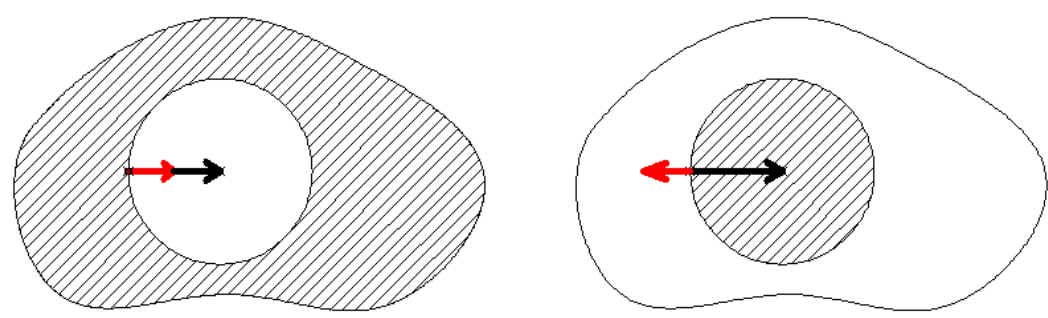

Figure (5): Difference between Hole and Shaft features

Once a hole or shaft features are recognized, they are added to the hole and shaft features dynamic lists. Hole or shaft feature object contains pointers to its underlying cylindrical face, center point, radius, axis and an ID number. The underling cylindrical surface of hole or shaft feature is finally sent to the sampling algorithm.

\section{Case studies:}

This section demonstrates the ability of the developed recognition algorithm. The algorithm can recognize different oriented planner faces as shown in figure (6). In addition to the recognition procedure, a planner face list is generated. A normal vector list is calculated for each planner face, Figure (7).

The algorithm is tested for a solid model containing a slot feature (planner face based feature) as shown in figure (8). For each recognized slot feature, its faces are ordered and stored as base face and side faces. Different three dimensional (3D) orientated slot features can also be detected by the developed recognition algorithm as shown in figure(9).
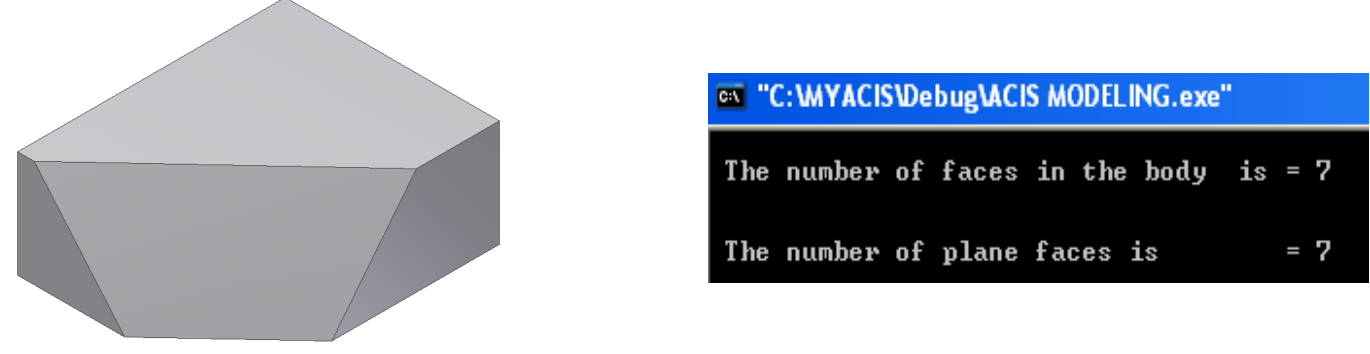

Figure (6): Recognition of inclined and oriented plan faces

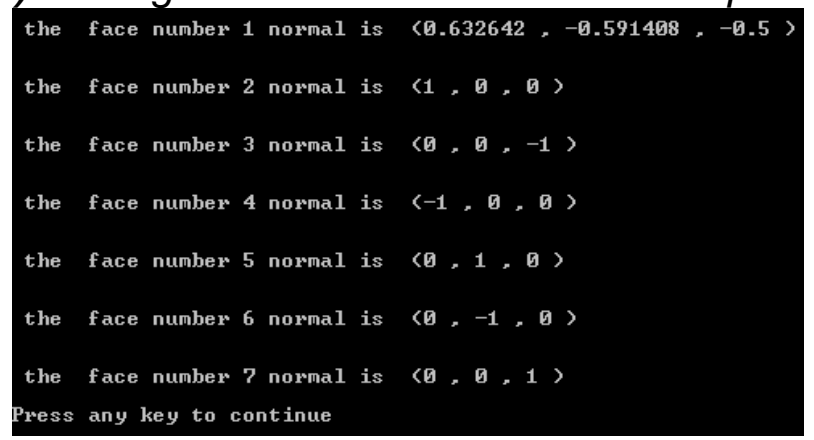

Figure (7): Plan faces normal vectors calculation process 

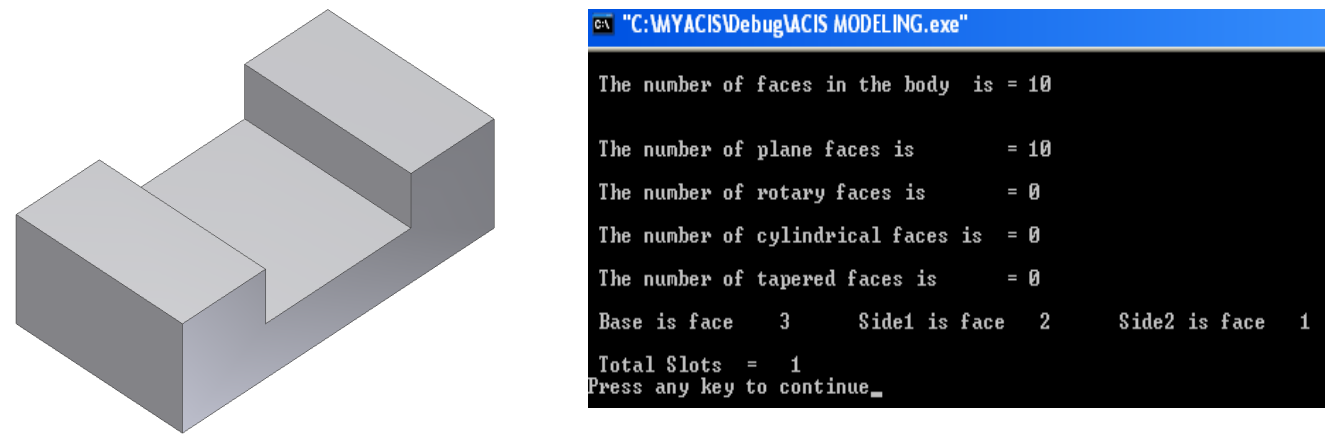

Figure (8): Slot feature Recognition
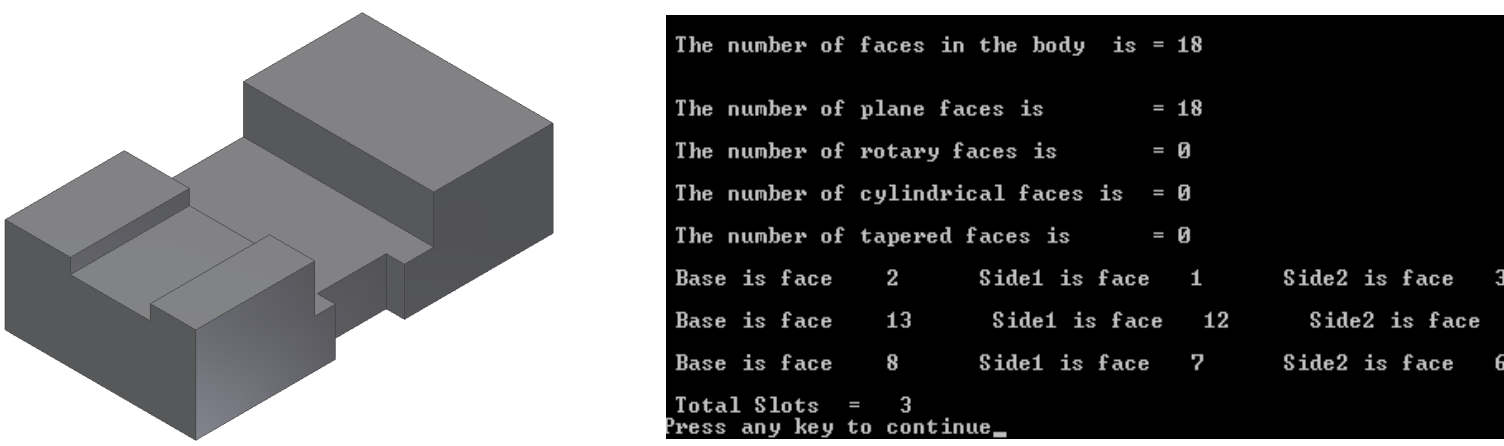

Figure (9): Recognition of different slot orientations

The developed feature recognition algorithm is also able to recognize through, blind and inclined hole features as shown in figures (10 - 12). Geometrical parameters associated with cylindrical hole are extracted as shown in figure (10). Complete/incomplete hole and shaft features can be recognized as shown in figure(13).
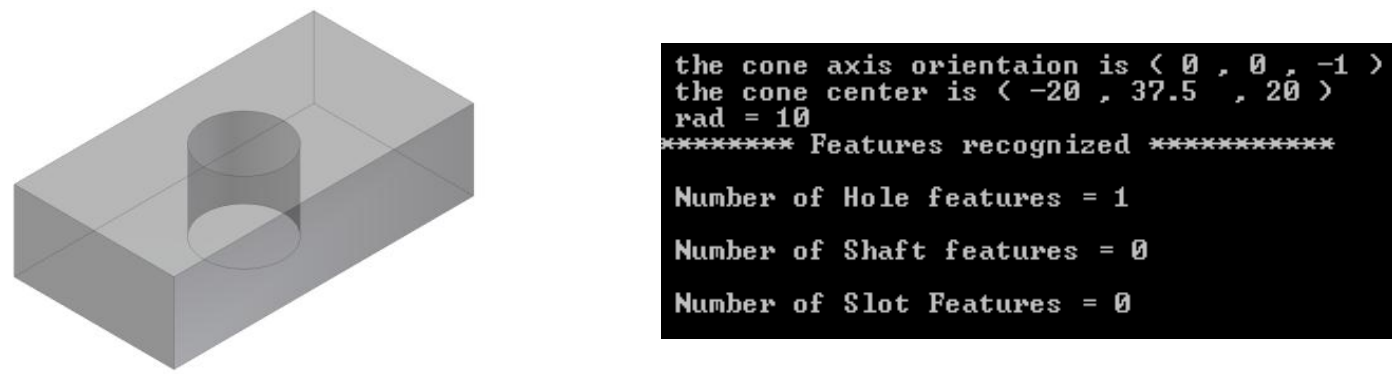

Figure (10): Recognition of a cylindrical through hole and its geometrical parameters
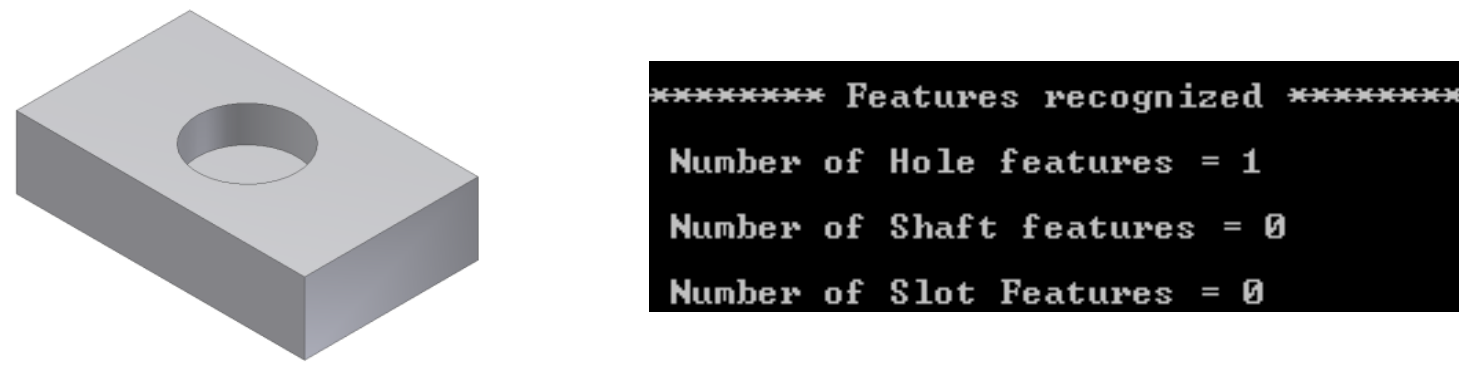

Figure (11): Recognition of blind hole feature 

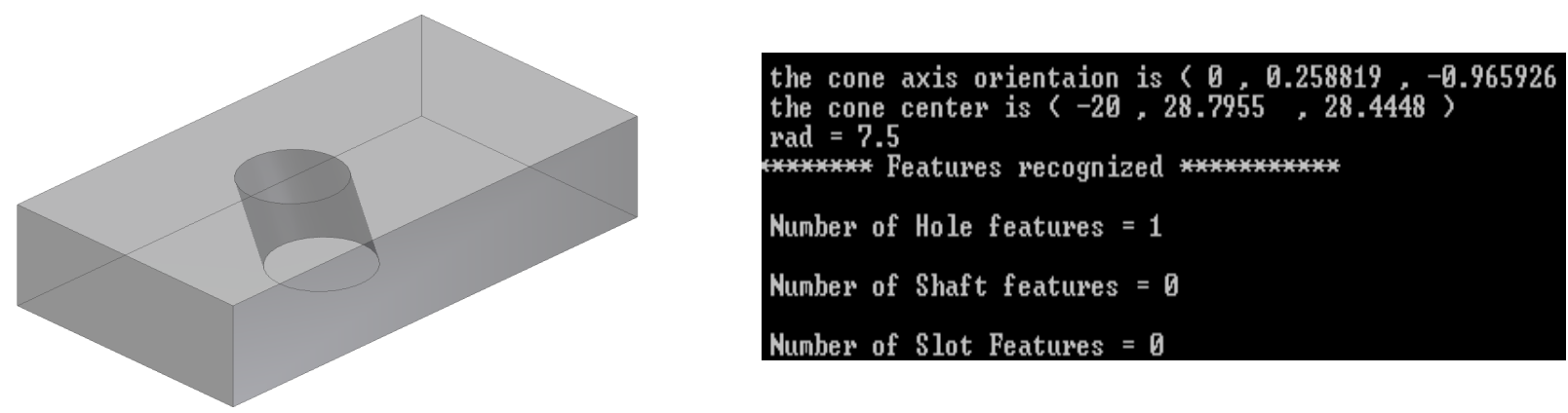

Figure (12): Recognition of inclined hole features
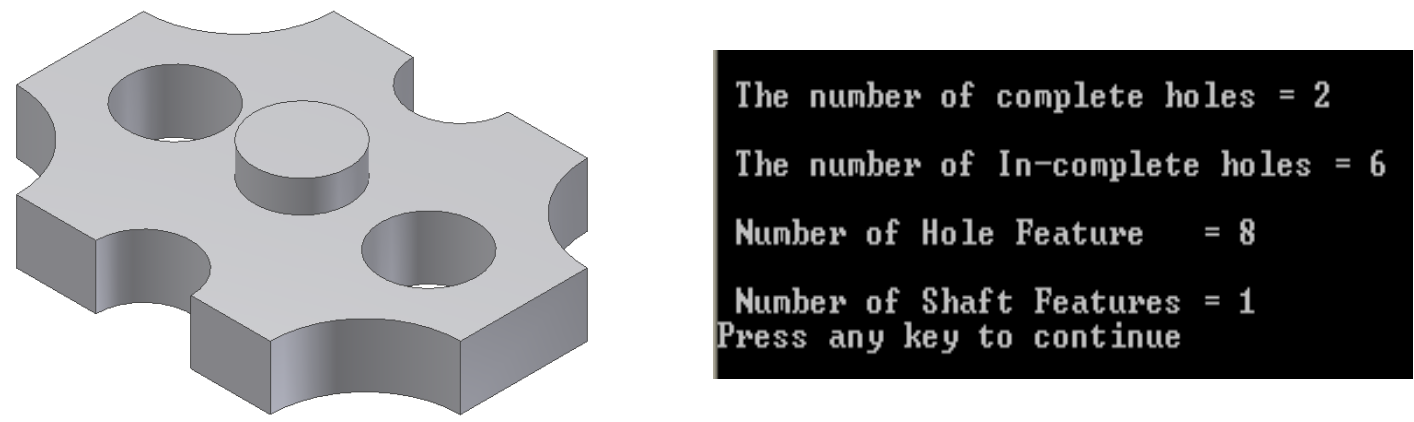

Figure (13): Recognition of complete/incomplete hole and shaft features

General case solid models are used to measure the ability of the recognition algorithm in recognizing prismatic features based on planner and cylindrical surfaces are shown in figures (14).
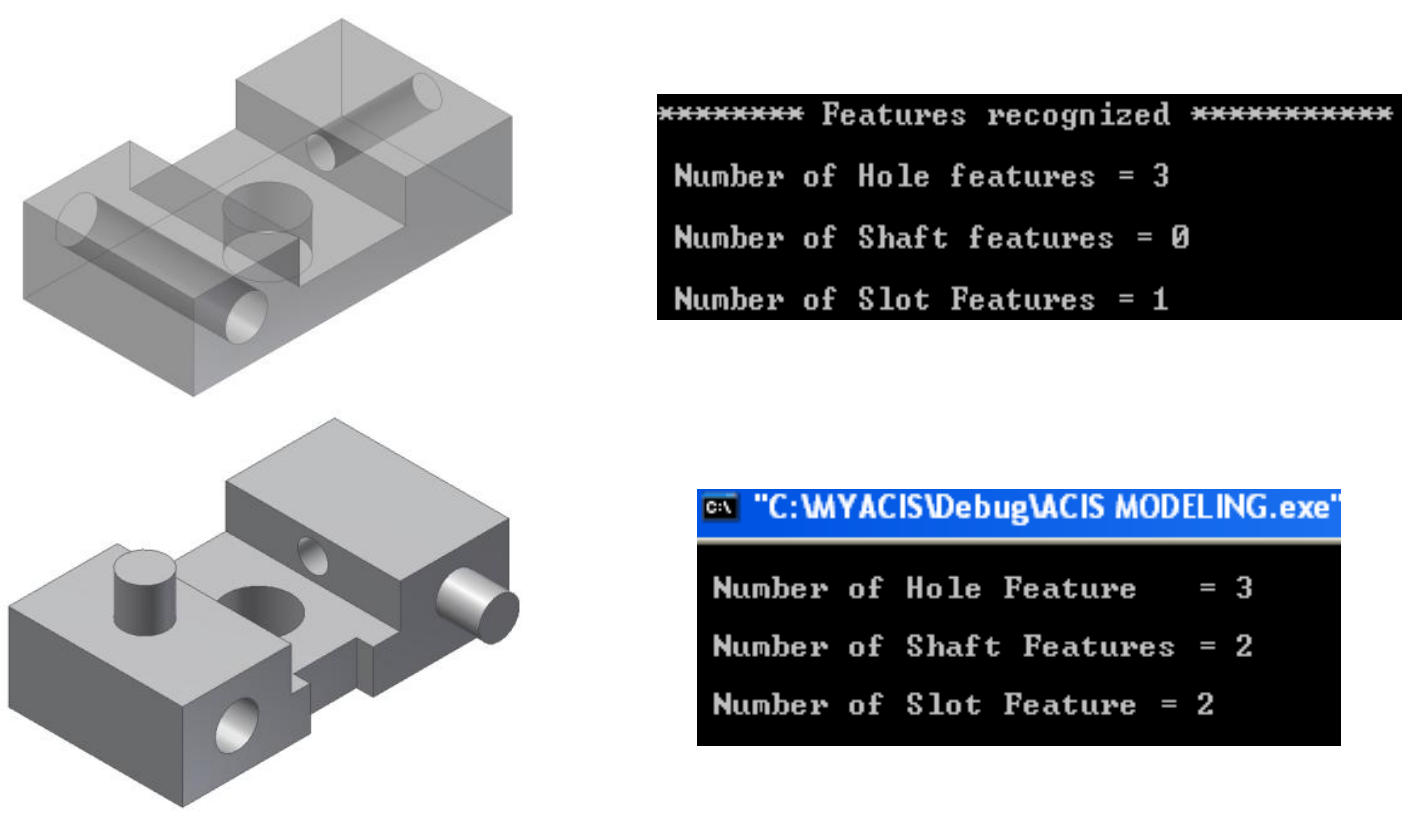

Figure (14): Recognition algorithm validation using a test solid models include different hole, shaft and slot features with multiple orientations 


\section{Conclusion:}

In this work, an automatic rule-based feature recognition system was developed. The developed algorithm is developed using ACIS geometric kernel and an object oriented programming techniques. The recognition algorithm deals with a B-rep solid model database that is given in SAT format. The recognition algorithm is capable of identifying prismatic features based on planner and cylindrical surfaces and having different orientations in 3D space. The algorithm was validated and tested using general cases that contain holes, shafts and slots in different orientations. This work is a part of an automatic inspection planning system for coordinate measuring machines.

\section{References:}

[1] A. M. Fikry, "Computer Aided Process Planning Using Feature Recognition For Milled Parts", Master, Military Technical Colllage, 1998

[2] M. R. Henderson and D. C. Anderson, "Computer Recognition and Extraction of Form Features: A CAD/CAM Link", Computers in Industry, 5 p.329-339, 1984.

[3] J. Han, M. Pratt and W. Regli, "Manufacturing feature recognition from solid models: a status report." IEEE Trans. Robotics and Automation, 16 (6), p.782-796, 2000.

[4] R. Abu and M. M. Tap, "Attribute Based Feature Recognition For Machining Features", Teknologi, 46 (A), p.87-103, 2007.

[5] D. Shikhare, "Feature-based Techniques for Handling Geometric Models", http://angelfire.lycos.com/space2/dineshshikhare/writings/features.pdf, 1999.

[6] S.-C. Liu, M. Gonzalez and J.-G. Chen, "Development of an automatic part feature extraction and classification system taking CAD data as input", Computers in Industry, 29 p.137-150, 1996.

[7] H. C. Lee, W. C. Jhee and H. S. Park, "Generative CAPP Through Projective Feature Recognition", Computers \& Industerial Engineering, 53 p.241-246, 2007.

[8] P. Chang and C. Chang, "An Integrated Artificial Intelligent Computer Aided Process Planning System", international Journal of Computer Integrated Manufacturing, 13 (6), p.483-497, 2000.

[9] J. J. Shah, "Assessment of Features Technology", Computer Aided Desgin, 23 (5), p.331-343, 1991.

[10] B. Babic, N. Nesic and Z. Miljkovic, "A review of automated feature recognition with rule-based pattern recognition", Computers in Industry, 59 p.321-337, 2008.

\section{Nomenclatures:}

CAPP

CAD

B-rep

CAM

OOP

CSG

IGES

STEP

2D

$3 \mathrm{D}$
Computer Aided Process Planning

Computer Aided design

Boundary representation

Computer Aided Manufacturing

Object Oriented Programming

Constructive solid geometry

Initial Graphics Exchange Specification

STandard for Exchange of Product data

Two dimensional

Three dimensional 